\title{
Magnetic Flux Leakage Course of Inner Defects and Its Detectable Depth
}

\author{
Jianbo Wu${ }^{1}$, Wenqiang $\mathrm{Wu}^{1}$, Erlong $\mathrm{Li}^{\mathrm{i}^{*}}$ (D) and Yihua Kang ${ }^{2}$
}

\begin{abstract}
As a promising non-destructive testing (NDT) method, magnetic flux leakage (MFL) testing has been widely used for steel structure inspection. However, MFL testing still faces a great challenge to detect inner defects. Existing MFL course researches mainly focus on surface-breaking defects while that of inner defects is overlooked. In the paper, MFL course of inner defects is investigated by building magnetic circuit models, performing numerical simulations, and conducting MFL experiments. It is found that the near-surface wall has an enhancing effect on the MFL course due to higher permeability of steel than that of air. Further, a high-sensitivity MFL testing method consisting of Helmholtz coil magnetization and induction coil with a high permeability core is proposed to increase the detectable depth of inner defects. Experimental results show that inner defects with buried depth up to $80.0 \mathrm{~mm}$ can be detected, suggesting that the proposed MFL method has the potential to detect deeply-buried defects and has a promising future in the field of NDT.
\end{abstract}

Keywords: Magnetic flux leakage (MFL) testing, Inner defect, Buried depth, Permeability

\section{Introduction}

As a powerful and highly efficient non-destructive testing (NDT) method, magnetic flux leakage (MFL) testing is conducted based on the physical phenomenon that a ferromagnetic specimen in a certain magnetization state will produce magnetic flux leakage if any discontinuities are presented in it [1]. Besides this, MFL is not affected by the attached non-ferromagnetic media around the specimens, such as when the surface has dirt or dust on it. Due to these advantages, MFL is especially suitable for steel structures inspection, such as bridge cables [2], pipelines [3, 4], rail tracks $[5,6]$, oil storage tank bottom $[7,8]$, steel ropes $[9,10]$, and steel pipes $[1,11]$.

MFL was firstly proposed to inspect magnetic penetration of ferrous materials in 1889 [12]. Until 1919, the MFL were truly applied for defects inspection by using a magnetizer to generate magnetic flux into the specimen, and induction coils to collect the leakage magnetic

\footnotetext{
${ }^{*}$ Correspondence: lierlg@scu.edu.cn

${ }^{1}$ School of Mechanical Engineering, Sichuan University, Chengdu 610065, China

Full list of author information is available at the end of the article
}

field caused by any defects [13]. Since then, the scientists mainly worked to propose and optimize the magnetizing $[14,15]$, sensing $[16,17]$, and signal processing methods [18]. Meanwhile, there has been also much researches on theoretical calculation of MFL distribution [19, 20], liftoff effect and high-speed effect [21-23]. Especially, with the rapid development of artificial intelligence, new data processing algorithm is used for defects recognition, classification and quantification [24-26].

It is well known MFL is sensitive to surface-breaking defects. However, when MFL is applied for deep-buried defects inspection, it faces a great challenge. In the MFL application, two common questions of MFL testing for inner defects must be figured out as follows: (1) why inner defects have a lower sensitivity than surface-breaking ones? (2) What is the detectable depth of MFL testing for inner defects? The first question is about MFL course of inner defects. Different from the surface-breaking defect, there is a ferromagnetic near-surface wall between the inner defect and the sensor. In the previous researches, the leakage mechanism analysis is mainly about the surface-breaking defects while that of inner defects is
Springer Open

(c) The Author(s) 2021. This article is licensed under a Creative Commons Attribution 4.0 International License, which permits use, sharing, adaptation, distribution and reproduction in any medium or format, as long as you give appropriate credit to the original author(s) and the source, provide a link to the Creative Commons licence, and indicate if changes were made. The images or other third party material in this article are included in the article's Creative Commons licence, unless indicated otherwise in a credit line to the material. If material is not included in the article's Creative Commons licence and your intended use is not permitted by statutory regulation or exceeds the permitted use, you will need to obtain permission directly from the copyright holder. To view a copy of this licence, visit http://creativeco mmons.org/licenses/by/4.0/. 
overlooked $[19,20]$. In this paper it is found that nearsurface wall has an enhancing effect on MFL course due to the higher permeability of steel than that of air, which will be analyzed in details. For the other question, figuring out the detectable depth limitation of inner defects is a key issue for MFL testing, especially for thick objects, such as thick-wall pipelines, large-diameter bridge cables, and rail tracks. The detectable depth determines whether the MFL method will be first choice or not. According to MFL inspection standard, MFL testing is usually applied to detect inner defects with a buried depth up to $20 \mathrm{~mm}$ [27], which is far from being able to meet the industrial inspection needs. Hence, a high-sensitivity MFL testing method for inner defects is in great need.

In this paper, in order to increase detectable depth of MFL for inner defects, MFL course of inner defects is firstly investigated, and then a high-sensitivity MFL testing method consisting of Helmholtz coil magnetization and induction coil with a high permeability core is proposed. The remaining sections are structured as: MFL course of inner defects is simply analyzed by building magnetic circuit models in Section 2; the simulation study of MFL distribution of inner defects are presented in Section 3; Section 4 experimentally studies MFL course of inner defects by analyzing signal responses; the detectable depth of inner defects is investigated by experiments in Section 5; a brief conclusion is given in Section 6.

\section{MFL Course Model of Inner Defects by Magnetic Circuit Models}

The principle of the MFL testing for inner defects is schematically illustrated in Figure 1(a). With a magnetizer, magnetic flux density $B_{0}$ is induced in a ferromagnetic specimen. Defects in the specimen will distort magnetic flux distribution and cause magnetic flux leak into the air. Above the specimen surface magnetic sensor are used to collect the leakage magnetic field, such as Hall sensor [17] and induction coil [28]. In the following part, three parameters are defined as: $d_{\mathrm{f}}$ (the distance between the sensor and the defect), $l_{\mathrm{d}}$ (the lift-off distance between the sensor and the specimen surface), $d_{\mathrm{b}}$ (the distance between the specimen surface and the defect, i.e., buried depth), and then we can get:

$$
d_{f}=l_{d}+d_{b} .
$$

In order to investigate the MFL course of inner defects, two different testing conditions are considered as follows:

(1) MFL course of defects D1 and D2: As displayed in Figure 1(a), comparing testing process for samesize D1 and D2, sensors S1 and S2 are placed above the specimen surface with the same lift-off distance of $l_{\mathrm{d}}$. No doubt, the D2 will generate a smaller signal response in the S2 than surface-breaking D1. In fact, there are two different influencing factors between the MFL courses of D1 and D2. There is a ferromagnetic wall between D2 and the S2 while there is none for surface-breaking D1. On the other hand, the distance $d_{\mathrm{f}}$ between the S2 and D2 is greater than that between S1 and D1. Hence, the reason why D2 has a lower sensitivity than D1 can hardly be attributed to the magnetic effect of the wall alone.

(2) MFL course of defects D2, D3 and D4: In previous studies, many authors investigated the MFL course of sub-surface defects using the defects D5, D6 and

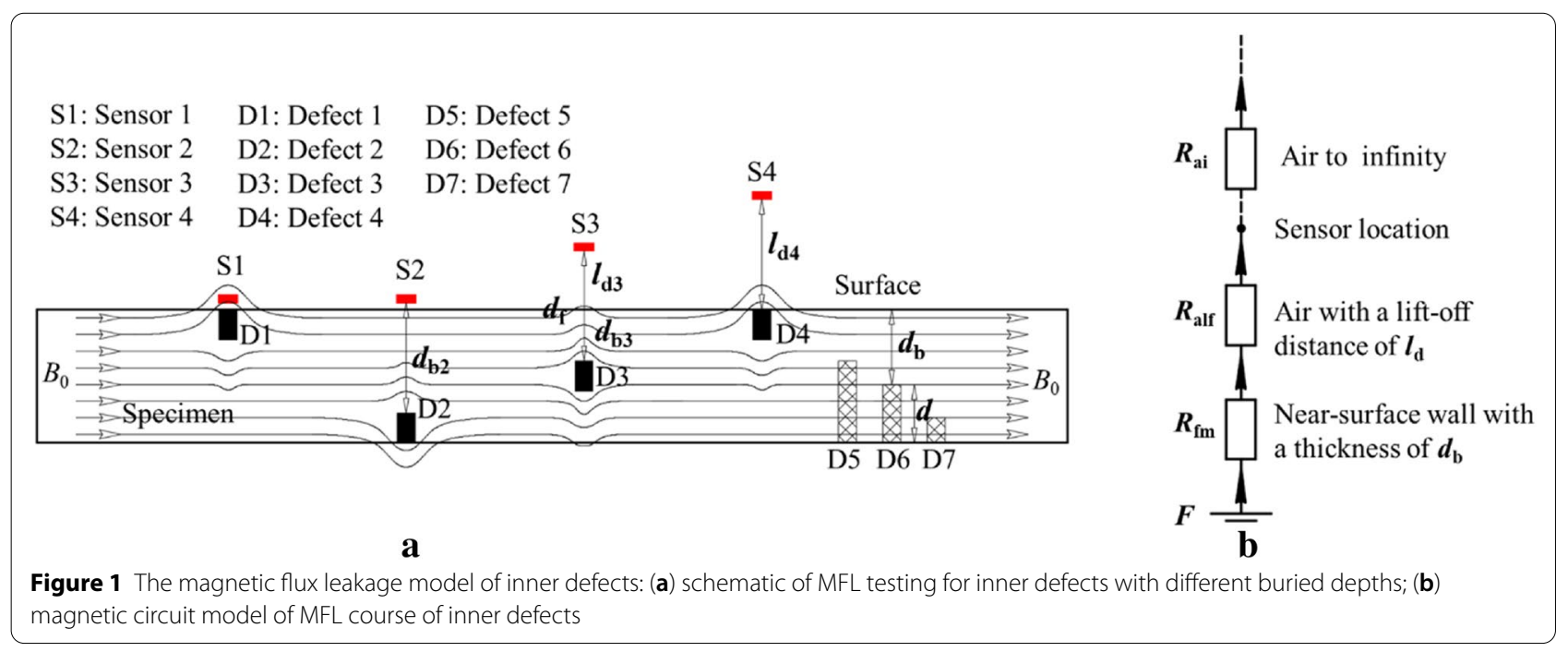


D7, as displayed in Figure 1(a) [29-31]. Although these three sub-surface defects have different buried depths $d_{\mathrm{b}}$, they also have different defect size (i.e., the defect depth $d$ ). Hence, the analysis model is flawed and the MFL signals are influenced by both the buried depth $d_{\mathrm{b}}$ and the defect size. In this paper, all the defects are designed to have the same size. Comparing testing process for same-size defects D2, D3 and D4, the distances $d_{\mathrm{f}}$ between three sensors and three defects have the same value. In this condition, only ferromagnetic wall factor will influence the sensitivity. With the proposed above analysis model, the MFL course of inner defects could be investigated.

The MFL course of inner defects is illustrated by the defect D3, as displayed in Figure 1(a). The S3 is placed above the specimen with a lift-off distance of $l_{\mathrm{d} 3}$, and the inner defect D3 is buried inside the specimen with a buried depth of $d_{\mathrm{b} 3}$. The distorted magnetic flux caused by the defect firstly passes through the near-surface wall with a thickness of $d_{\mathrm{b} 3}$ (i.e., the buried depth of the defect), then leaks into the air, and finally reaches the sensor location.

Based on Hopkinson's law [32], the magnetic flux leakage course of inner defects is simply built by magnetic circuit models, as displayed in Figure 1(b). From the inner defect to the sensor location, there are ferromagnetic wall and non-ferromagnetic air, and their magnetic reluctance are $R_{\mathrm{fm}}$ and $R_{\mathrm{alf}}$, respectively. From the sensor location to infinite far place, the magnetic reluctance of the air is $R_{\mathrm{a} 1}$. Then, we can get

$$
\phi=\frac{F}{R_{\mathrm{fm}}+R_{\mathrm{alf}}+R_{a 1}},
$$

where $F$ denotes the magnetomotive force depending on the defect size and magnetization intensity; $\phi$ denotes the leakage magnetic flux. In the model all defects have the same size and the magnetization intensity is fixed, hence the $F$ is constant.

The magnetic reluctance is expressed as follows:

$$
R=\frac{h}{\mu A},
$$

where $h$ is the length of material, $\mu$ is the permeability of the material, and $A$ is the cross-sectional area. Based on Eqs. (2) and (3), the leakage magnetic flux can be expressed as follows:

$$
\phi=\frac{F}{\frac{d_{\mathrm{b}}}{\mu_{\mathrm{fm}} A}+\frac{l_{\mathrm{d}}}{\mu_{\mathrm{a}} A}+\frac{h_{\mathrm{a}}}{\mu_{\mathrm{a}} A}},
$$

where $\mu_{\mathrm{fm}}$ and $\mu_{\mathrm{a}}$ denote the permeability of the specimen and air, respectively. In order to analyze the magnetic effect of near-surface wall, the distance between the defect and the sensor is set as a fixed value of $d_{\mathrm{f}}$, then, the lift-off distance $l_{\mathrm{d}}$ can be expressed as follows:

$$
l_{\mathrm{d}}=d_{\mathrm{f}}-d_{\mathrm{b}} .
$$

Finally, the relationship between leakage magnetic flux $\phi$ and buried depth $d_{\mathrm{b}}$ can be simplified as follows:

$$
\phi=\frac{F}{\frac{d_{\mathrm{b}}}{\mu_{\mathrm{fm}} A}+\frac{d_{\mathrm{f}}-d_{\mathrm{b}}}{\mu_{\mathrm{a}} A}+\frac{h_{\mathrm{a} 1}}{\mu_{\mathrm{a}} A}}=\frac{F}{\frac{d_{\mathrm{f}}}{\mu_{\mathrm{a}} A}-\frac{d_{\mathrm{b}}\left(\mu_{\mathrm{fm}}-\mu_{\mathrm{a}}\right)}{\mu_{\mathrm{fm}} \mu_{\mathrm{a}} A}+\frac{h_{\mathrm{a} 1}}{\mu_{\mathrm{a}} A}} .
$$

From Eq. (6), it can be seen with the same distance $d_{\mathrm{f}}$, the leakage magnetic flux $\phi$ and the buried depth $d_{\mathrm{b}}$ has a positive correlation. That is to say, the deepest-buried D2 will generate the greatest MFL signal response in the sensor among D2, D3 and D4. Hence, it could be concluded that the near-surface wall has an enhancing effect on the MFL course of inner defects due to the higher permeability of steel than those of air.

\section{MFL Course Simulation of Inner Defects}

Efficient magnetization is the basis to realize MFL inspection for inner defects. There are three types of MFL methods using different magnetization modes, namely, pulsed MFL [29], alternating current (AC) MFL $[30,31]$ and direct current (DC) MFL. However, for deepburied defects inspection, the former two magnetization methods are unsuitable due to skin effect of alternating magnetic field. In contrast, with large DC magnetizing intensity, DC MFL can magnetize thick specimens into saturation status to maximize leakage magnetic field of deep-buried defects. In this paper, a Helmholtz coil magnetizer are proposed to produce a strong and uniform magnetizing field for thick specimen. In order to validate the MFL course of inner defects, numerical simulations are conducted by Ansoft Maxwell with the magnetostatic solver. A two-dimensional finite element method (FEM) model is built as shown in Figure 2. The parameters of the Helmholtz coils are as follows: the height of the coil is $100 \mathrm{~mm}$, the thickness of the coil is $100 \mathrm{~mm}$, the distance between the coil inner surface and the specimen surface is $20 \mathrm{~mm}$, and the distance between the two coils is $100 \mathrm{~mm}$, respectively. An inner defect (width: $4.0 \mathrm{~mm}$; height: $6.0 \mathrm{~mm}$ ) is made inside a steel plate (length: $1000.0 \mathrm{~mm}$; thickness: $100.0 \mathrm{~mm}$; Relative permeability: $\mu-H$ curve of ASTM A29). The magnetization current density in each coil is set as $6 \times 10^{6} \mathrm{~A} / \mathrm{mm}^{2}$ to magnetize the thick plate into saturation state.

Figure 3 shows the distorted magnetic field distribution caused by inner defects with buried depths of $0.0 \mathrm{~mm}$, 


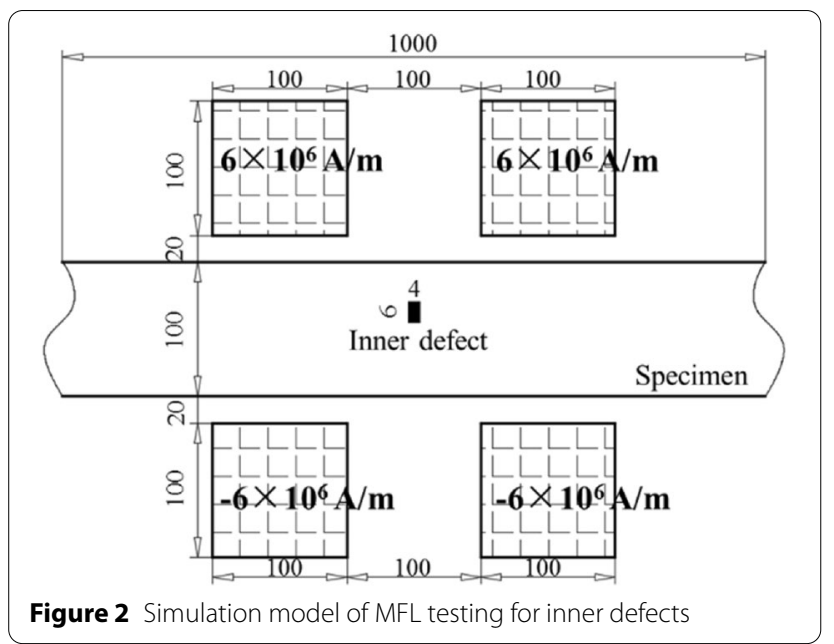

$2.0 \mathrm{~mm}, 5.0 \mathrm{~mm}$ and $8.0 \mathrm{~mm}$, respectively. The rainbow lines above the specimen surface display the leakage magnetic flux distribution $B_{\mathrm{MFL}}$. It can be seen different $B_{\mathrm{MFL}}$ distributions are generated by these defects with different buried depths. In addition, with the buried depth increasing, leakage magnetic flux density is decreasing.

In previous MFL distribution studies, the $B_{\mathrm{MFL}}$ above the specimen surface is the main concern while the magnetic flux distribution inside the specimen has been ignored. In order to investigate the magnetic effect of near-surface wall on the MFL course, the magnetic flux distributions inside the specimen are simulated as indicated by rainbow color map. It can be seen that the magnetic flux in the near-surface wall is distorted greatly, and that the $B_{\mathrm{MFL}}$ above the specimen is actually generated by the distorted magnetic flux in the near-surface wall. As displayed in Figure 3(a), along the collecting line $L_{\mathrm{c}}$ (length: $30.0 \mathrm{~mm}$; lift-off distance $l_{\mathrm{d}}: 1.0 \mathrm{~mm}$ ) the normal component of magnetic field above the surface-breaking defect is collected and indicated by red solid line as shown in Figure 4. It can be seen that the signal manifest itself an odd function with the sharpest gradient in the center. In addition, there is a baseline-shift phenomenon of the testing signal. Specifically, the left side of the signal has a positive baseline shift while the right side is characterized by a negative one, which will result in a rough evaluation.

As schematically illustrated in Figure 5, the measured magnetic field (defined as $B_{\mathrm{m} \text {-defect }}$ ) at the sensor location

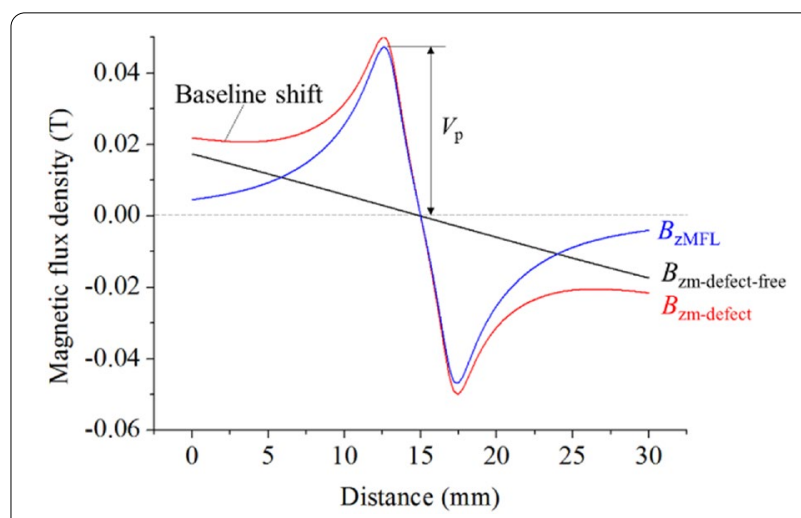

Figure 4 The normal components of magnetic field along the collecting line $L_{c}$

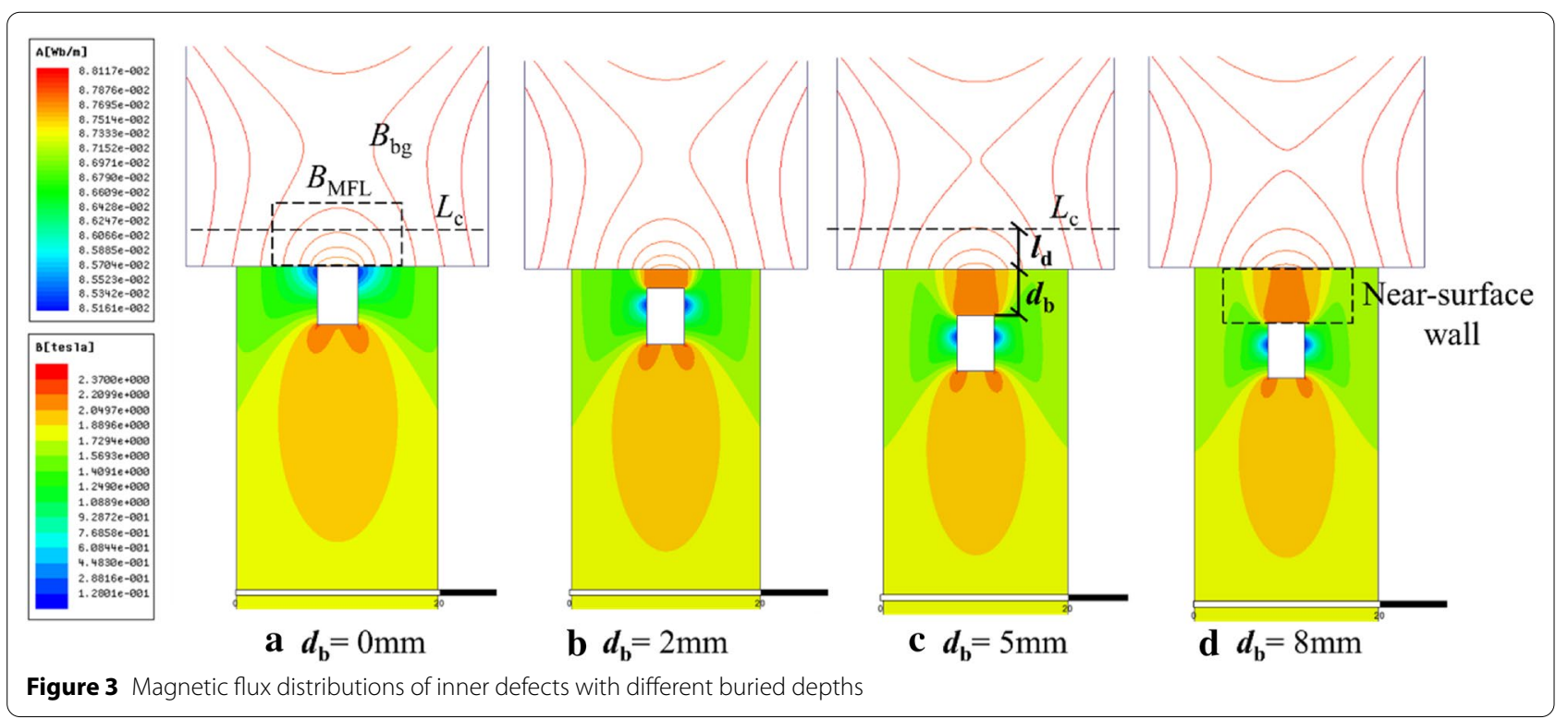




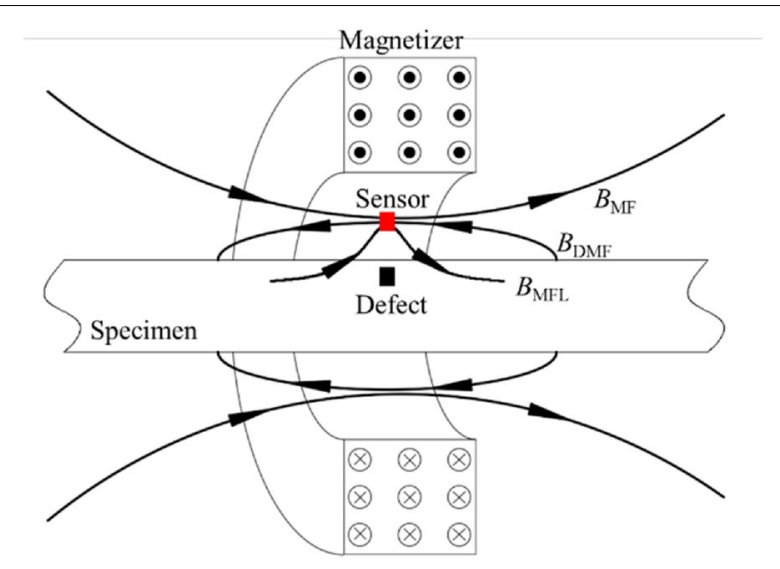

Figure 5 Measuring principle in the MFL testing

is actually composed of leakage magnetic field generated by the defects (defined as $B_{\mathrm{MFL}}$ ), magnetizing field generated by the magnetizer (defined as $B_{\mathrm{mg}}$ ), and the demagnetizing field caused by the specimen (defined as $B_{\mathrm{dmg}}$ ). Background magnetic field (defined as $B_{\mathrm{bk}}$ ) is composed of the $B_{\mathrm{mg}}$ and the $B_{\mathrm{dmg}}$. The relationship can be expressed by follows [33]:

$$
B_{\mathrm{m} \text {-defect }}=B_{\mathrm{MFL}}+B_{\mathrm{mg}}+B_{\mathrm{dmg}}=B_{\mathrm{MFL}}+B_{\mathrm{bk}}
$$

where $B_{\mathrm{mg}}$ and $B_{\mathrm{dmg}}$ are determined by the magnetizing field distribution and the specimen structure. The $B_{\mathrm{bk}}$ is normally constant under the same conditions.

When there is no defect in the specimen, the measured magnetic field $B_{\mathrm{m} \text {-defect-free }}$ contains only $B_{\mathrm{bk}}$ :

$$
B_{\mathrm{m} \text {-defect }- \text { free }}=B_{\mathrm{bk}} \text {. }
$$

Then, $B_{\mathrm{MFL}}$ can be obtained from Eq. (7) by subtracting Eq. (8):

$$
B_{\mathrm{MFL}}=B_{\mathrm{m} \text {-defect }}-B_{\mathrm{m} \text { - defect }- \text { free. }}
$$

In order to eliminate the $B_{\mathrm{bk}}$ and observe the $B_{\mathrm{MFL}}$ alone, a defect-free specimen under the same condition is simulated. The normal component of the $B_{\mathrm{m} \text {-defect-free }}$ (defined as $B_{\text {zm-defect-free }}$ ) is calculated and indicated by black solid line in Figure 4 . It can be seen that the $B_{\mathrm{zm} \text { - }}$ defect-free shows a slash with a negative slope. After subtraction, the normal component of $B_{\mathrm{MFL}}$ (defined as $\left.B_{\mathrm{zMFL}}\right)$ is obtained as indicated by the blue solid line, and the baseline shift phenomenon disappears. In the following simulation, the $B_{\mathrm{bk}}$ will be eliminated by the same subtraction method, and the peak amplitude of $B_{\mathrm{zMFL}}$ (defined as $V_{\mathrm{p}}$ ) will be used to compare the sensitivity as shown in Figure 4.

Further, ten defects with different buried depths from $0.0 \mathrm{~mm}$ to $10.0 \mathrm{~mm}$ are simulated. Along the collecting line $L_{\mathrm{c}}$ at the same lift-off distance $l_{\mathrm{d}}$ of $1.0 \mathrm{~mm}$, the
$B_{\mathrm{zMFL}}$ are collected and plotted, as shown in Figure 6. It can be seen at the same lift-off distance $l_{\mathrm{d}}$ of $1.0 \mathrm{~mm}$, the deeper-buried defect generates a weaker signal response. Then, their peak amplitudes of $B_{\mathrm{zMFL}}\left(V_{\mathrm{p}}\right)$ are extracted and indicated by dotted blue line in Figure 7 , which shown a descending trend with the increasing buried depth. Further, along other collecting lines $L_{\mathrm{c}}$ with the $l_{\mathrm{d}}$ varying from 2.0 to $9.0 \mathrm{~mm}$, the $V_{\mathrm{p}}$ of the ten defects are extracted and displayed in Figure 7. It can be seen that the deeper-buried inner defect has a lower sensitivity at the same lift-off distance $l_{\mathrm{d}}$.

The above simulation is used to illustrate different MFL course of defects D1 and D2, as shown in Figure 1(a). As we know, there are two different influencing factors, i.e., the distance between the sensor and the inner defect, and the magnetic effect of the near-surface wall, which must be analyzed separately.

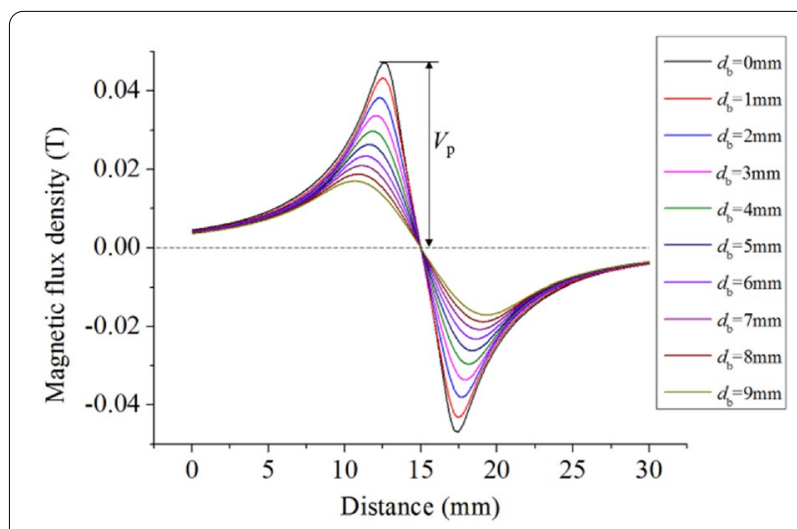

Figure 6 The $B_{\mathrm{ZMFL}}$ of ten inner defects at the same lift-off distance $l_{d}$ of $1.0 \mathrm{~mm}$

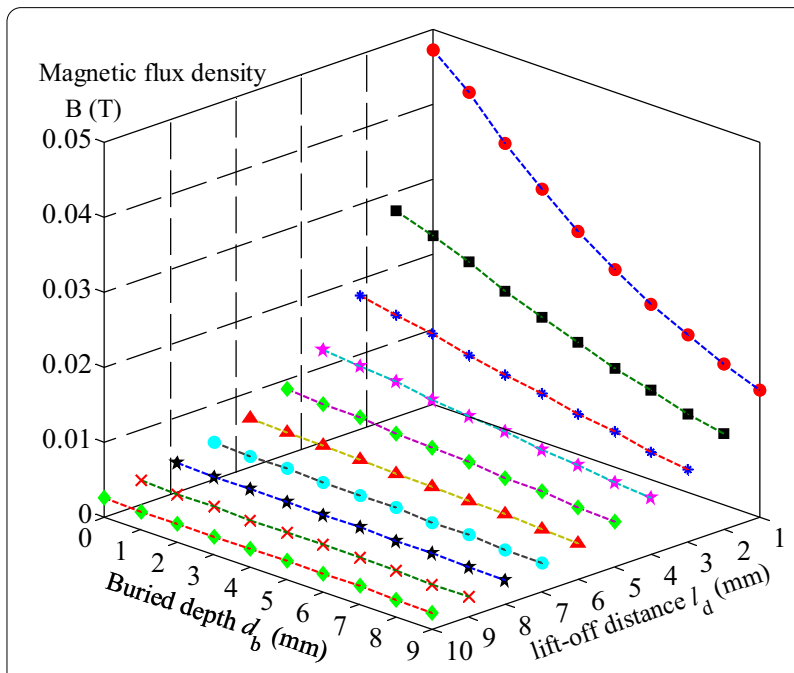

Figure 7 The $V_{p}$ of ten inner defects with the sensor locating at the same lift-off distance $I_{d}$ 


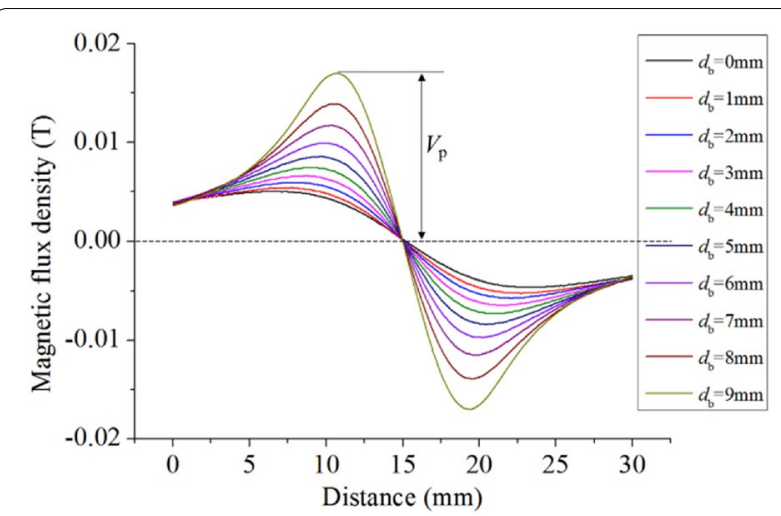

Figure 8 The $B_{\text {zMFL }}$ of ten inner defects with same fixed distance $d_{f}$ of $10.0 \mathrm{~mm}$

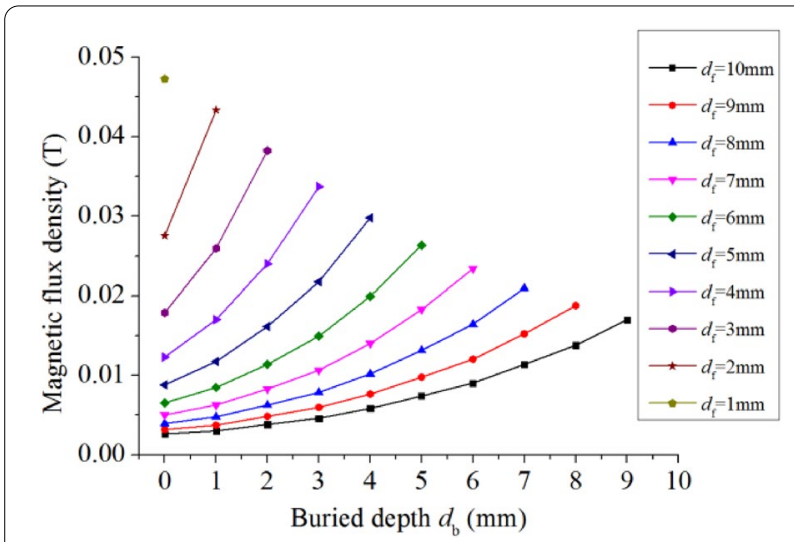

Figure 9 The $V_{p}$ of ten inner defects with the distance $d_{f}$ from 1.0 to $10.0 \mathrm{~mm}$

In order to analyze the magnetic effect of near-surface wall, the distance between the defect and the sensor is set as a fixed value of $d_{\mathrm{f}}$ as illustrated by the Defects D2, D3 and D4 in Figure 1(a). Firstly, setting the fixed distance $d_{\mathrm{f}}$ of $10.0 \mathrm{~mm}$, the $B_{\mathrm{zMFL}}$ of ten inner defects are collected and plotted in Figure 8. It can be seen that when the $d_{\mathrm{f}}$ is set as a fixed value, the deeper-buried defect generates a stronger signal response. Then, their $V_{\mathrm{p}}$ are extracted and indicated by black solid line in Figure 9, which shown an increasing trend with the buried depth increasing. Further, other $d_{\mathrm{f}}$ (from $1.0 \mathrm{~mm}$ to $9.0 \mathrm{~mm}$ ) are simulated, as shown in Figure 9. The results validate that the nearsurface wall actually has an enhancing effect on the MFL course of inner defect. The simulation results match the theoretical model well as expressed by Eq. (5).

\section{MFL Course Experiment of Inner Defects}

In previous MFL experimental studies of inner defects, the specimen is usually designed with sub-surface defects [27-29], which cannot present inner defects with the

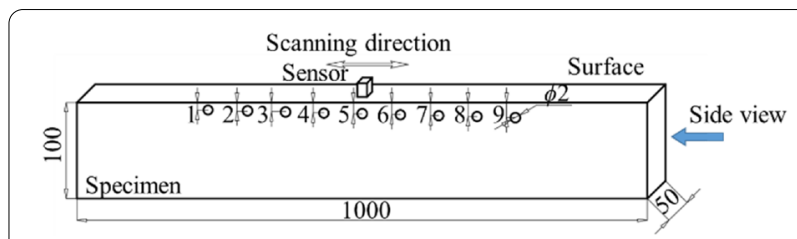

Figure 10 Schematic diagram of MFL testing for inner defects with different buried depths

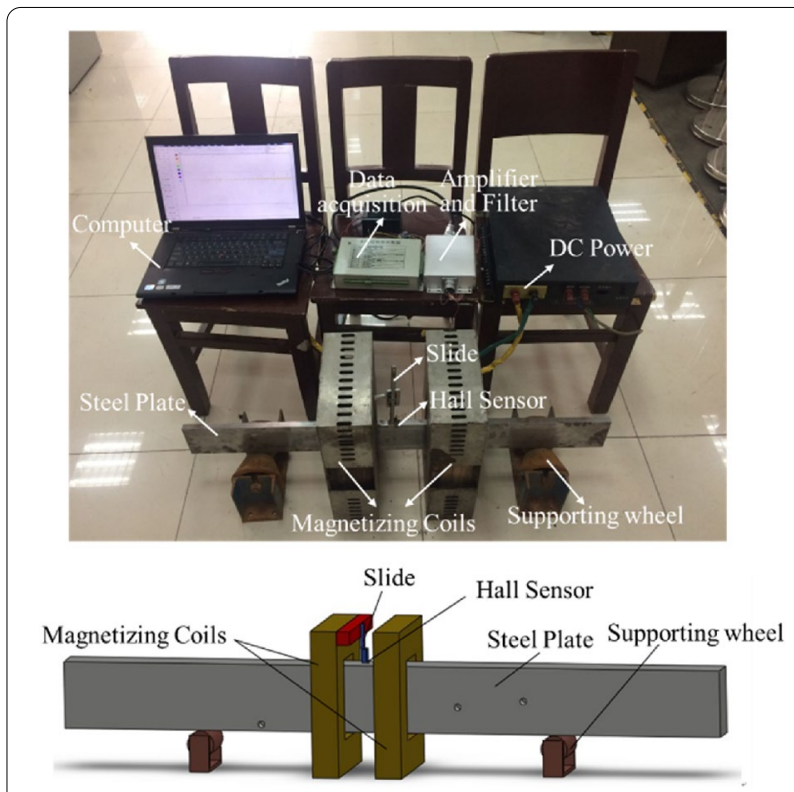

Figure 11 MFL experimental setup for inner defects

same size but different buried depths. In order to investigate the MFL course of inner defects, a specimen with same-size inner defects is proposed and designed, as displayed in Figure 10. Nine same-size blind holes (diameter: $2.0 \mathrm{~mm}$; depth: $20.0 \mathrm{~mm}$ ) with different buried depths (from $1.0 \mathrm{~mm}$ to $9.0 \mathrm{~mm}$ ) are manufactured in the side-surface of a steel plate (length: $1000.0 \mathrm{~mm}$; width: $50.0 \mathrm{~mm}$; height: $100.0 \mathrm{~mm}$ ). Meanwhile, a sensor is placed on the top surface to scan the defects. In this way, MFL courses of different inner defects can be easily compared under the same inspection condition. The experimental setup is built as pictured in Figure 11. The number of square Helmholtz coil turns is 1000. A DC current with amplitude of $10 \mathrm{~A}$ is applied to Helmholtz coils to magnetize the steel plate into saturation status. Above the specimen top surface, Hall sensor (Allegro 1302) commonly used in industry is mounted onto a guide track, which can adjust the lift-off distance. The inspected plate is driven to move by supporting wheels with motors at a fixed speed of $50.0 \mathrm{~mm} / \mathrm{s}$. When the plate passes through the Helmholtz coils, the normal 
components of leakage magnetic field will be collected by the Hall sensor; then, the collected signal will be processed by an amplifier and then a filter for signal conditioning; further, the processed signal will go into the data acquisition device for changing analog signal to the digital signal; finally the digital signal will be stored and analyzed by the computer.

When Hall sensor scans at a lift-off distance $l_{\mathrm{d}}$ of $1.0 \mathrm{~mm}$, normal MFL signals of the blind hole with buried depth $d_{\mathrm{b}}$ of $1.0 \mathrm{~mm}$ is collected, as shown in Figure 12. It can be seen the signal manifest itself an odd function with the sharpest gradient in the center, which is accordant with the simulation results as shown in Figure 4. Besides, there is no signal baseline shift phenomenon. In the MFL scanning process, the sensor is fixed at the middle of the two Helmholtz coils, and there is no relative motion between magnetizing field and Hall sensor. Hence, the normal component of the background magnetic field at the sensor location is constant and closed to zero.

In the experimental scheme, an additional question needs to be confirmed. As displayed in Figure 13(a), since blind holes are manufactured in the side-surface of the specimen, there are two possible leakage paths from the blind hole to the sensor location. Though path (1), the distorted magnetic field passes to Hall sensor location though the side air space. In this situation, the leakage course will be not influenced by the near-surface wall, which is different from the MFL course of inner defects indicated by path (2). In order to confirm the true leaking path, contrast experiments are conducted. Firstly, a steel plate with a thickness of $20.0 \mathrm{~mm}$ is used to cover the blind holes, as displayed in Figure 13(b). In this condition, the distorted magnetic flux of inner defects can only leak though the path (2). Then, as displayed in

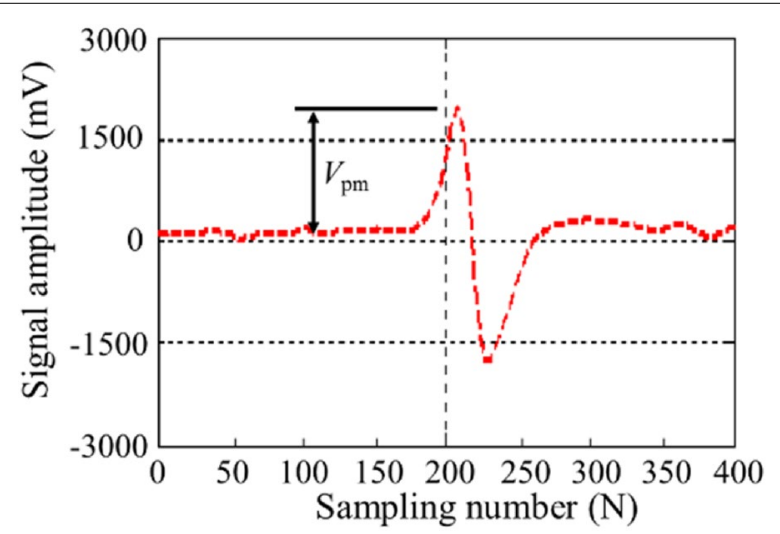

Figure 12 The normal component of leakage magnetic field of inner defect with buried depth $d_{\mathrm{b}}$ of $1.0 \mathrm{~mm}$ and the sensor is placed at a lift-off distance $I_{d}$ of $1.0 \mathrm{~mm}$

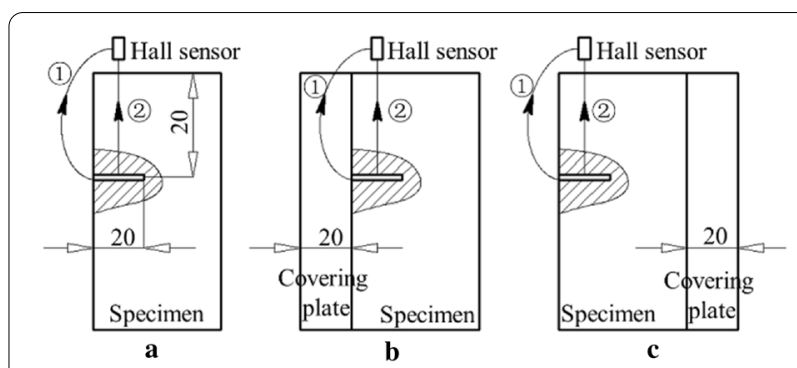

Figure 13 The possible leakage paths in the MFL experiment (Side view in Figure 10)

Figure 13(c), the same covering plate is placed on the other side of the specimen to obtain the same magnetization status. Experimental results show that the same inner defect in Figure 13(b) and Figure 13(c) generates a same signal response. Hence, it can be concluded that the distorted magnetic field of the inner defect leaks though path (2) due to the high permeability of the near-surface wall. In the following experiments, the scheme displayed in the Figure 13(a) will be used to investigate the magnetic effect of the near-surface wall.

Similar with the simulations, the peak values of the tested signals $\left(V_{\mathrm{pm}}\right)$ are used to investigate the sensitivity, as defined in Figure 12. Firstly, nine blind holes are scanned by Hall sensor at the same lift-off distance $l_{\mathrm{d}}$ of $1.0 \mathrm{~mm}$, and their $V_{\mathrm{pm}}$ are extracted and indicated by dotted blue line, as displayed in Figure 14. With the buried depth of blind holes increasing, their MFL signal amplitudes present a declining trend. Further, the $V_{\mathrm{pm}}$ at other lift-off distance $l_{\mathrm{d}}$ (from $2.0 \mathrm{~mm}$ to $9.0 \mathrm{~mm}$ ) are collected and extracted, as shown in Figure 15. The experimental

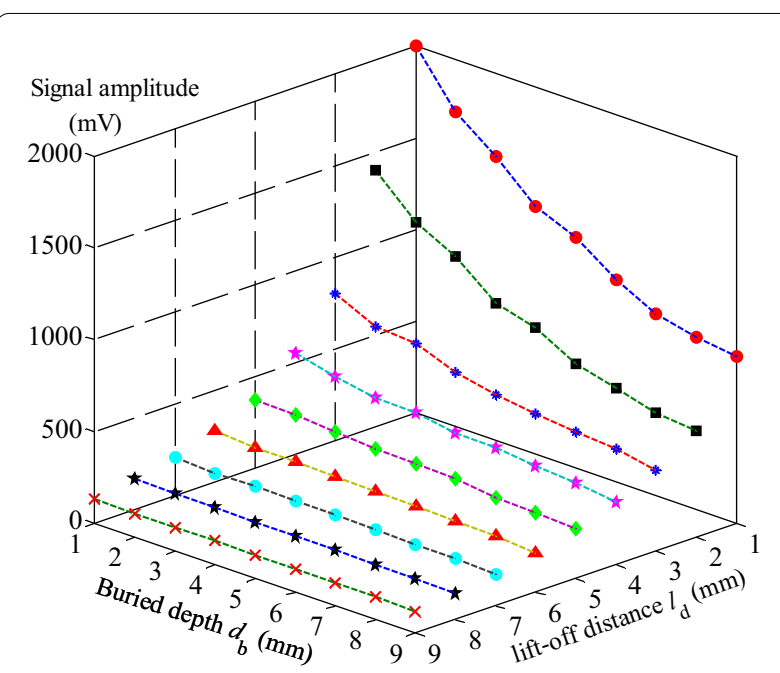

Figure 14 The $V_{p m}$ of the nine inner defects at the same lift-off distance of $I_{d}$ from the sensor to the specimen surface 


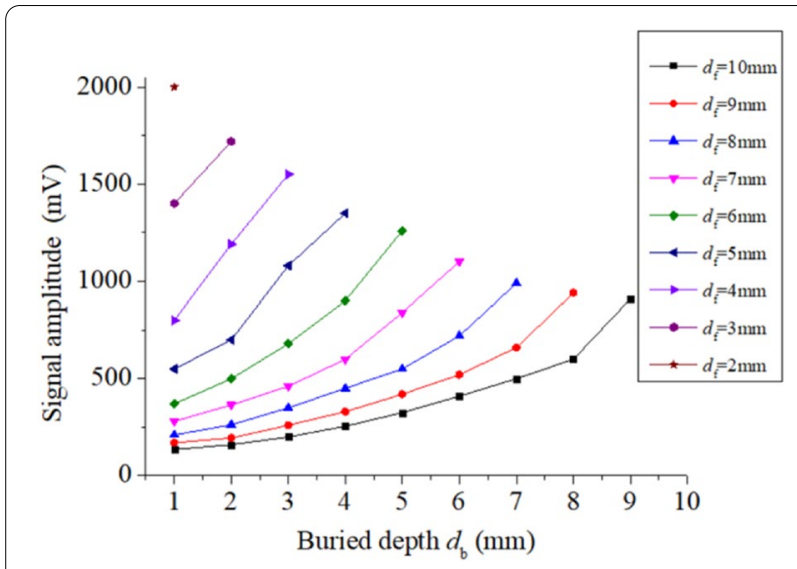

Figure 15 The $V_{\mathrm{pm}}$ of the nine inner defects with the same fixed distance $d_{f}$

results validate that the deeper-buried inner defect has a lower sensitivity, which matches simulation results well as displayed in Figure 7.

However, the above experiments cannot explain the MFL course of the inner defects, since there are two variables discussed above, including different distances $d_{\mathrm{f}}$ from the sensor to blind holes, and magnetic effect of the near-surface wall. In order to separately analyze the magnetic effect, the distance $d_{\mathrm{f}}$ between the sensor and blind hole is set as a fixed value. Firstly, the $d_{\mathrm{f}}$ is set as $10.0 \mathrm{~mm}$ by changing the lift-off distance $l_{\mathrm{d}}$ of Hall sensor. Specifically, for nine blind holes with buried depths from $1.0 \mathrm{~mm}$ to $9.0 \mathrm{~mm}$, the Hall sensor is placed at the lift-off distances $l_{\mathrm{d}}$ from $9.0 \mathrm{~mm}$ to $1.0 \mathrm{~mm}$, respectively. The extracted $V_{\mathrm{pm}}$ are shown by black solid line in Figure 15 , which shows that with the same $d_{\mathrm{f}}$ of $10.0 \mathrm{~mm}$, the deeper-buried defect generates a greater signal response. Further, the nine blind holes are scanned with other $d_{\mathrm{f}}$ (from $2.0 \mathrm{~mm}$ to $9.0 \mathrm{~mm}$ ) and the extracted $V_{\mathrm{pm}}$ are displayed in Figure 15. The experimental results are accordant with simulation results displayed in Figure 9 and the theoretical model described by Eq. (5). It can be proven that the near-surface wall has an enhancing effect on the MFL course due to the higher permeability of steel than those of air.

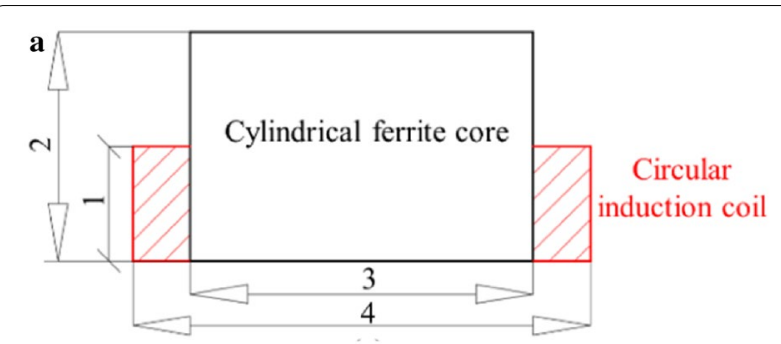

b

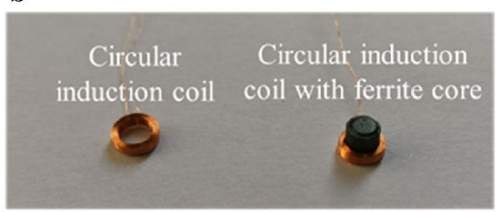

Figure 16 Circular induction coil with ferrite core: (a) structure of the proposed sensor; (b) picture of the proposed sensor

\section{Detectable Depth of MFL for Inner Defects}

Figuring out the detectable depth limitation of inner defects is a key issue to MFL application. The detectable depth determines whether the MFL method will be first choice or not. Efficient magnetization is the basis for deep-buried defects inspection. Especially for specimens with great thickness, strong magnetization is needed to excite deep-buried defects to produce a detectable leakage magnetic field. On the other hand, a high-sensitivity sensing method is needed to efficiently collect the weak leakage magnetic field of inner defects. In the above experiments, it was found that Hall sensor commonly used in industry cannot detect inner defects with buried depth greater than $20.0 \mathrm{~mm}$. In the traditional sensing process, the MFL field is distributed freely and its density depends on defect size and magnetization intensity, which can be named by a "passive sensing method", leading to a limited sensitivity of inner defects. In this paper, in addition to the proposed Helmholtz coil magnetizing method, an induction coil with a high permeability core is proposed and developed to increase the detectable depth, which is an active sensing method. As displayed in Figure 16, the proposed active sensor consists of a circular induction coil and a cylindrical ferrite core. Specifically, the ferrite core (Diameter: $3.0 \mathrm{~mm}$; Height: $2.0 \mathrm{~mm}$ ) is wound around by the insert coil (External diameter: $4.0 \mathrm{~mm}$; Internal diameter: $3.0 \mathrm{~mm}$; Height: 

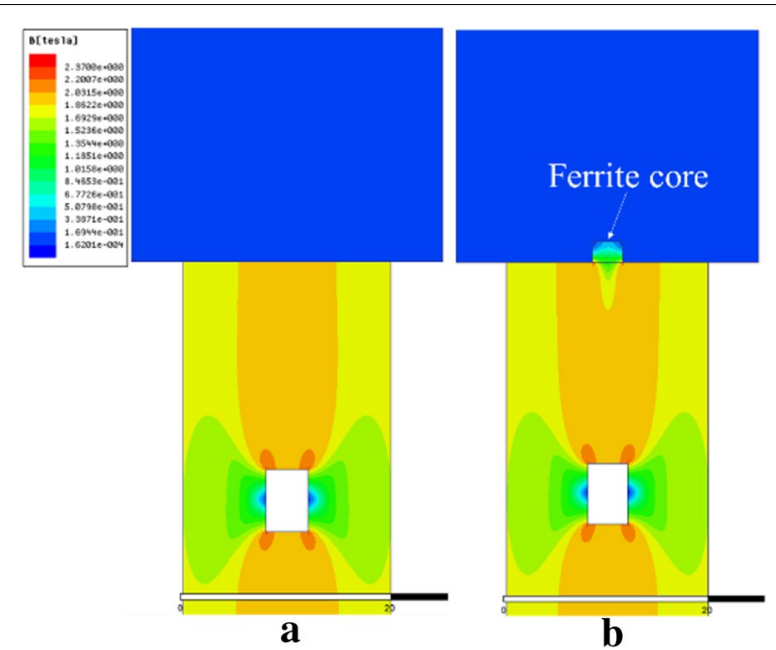

Figure 17 The magnetic flux density distribution: (a) traditional passive sensing method; (b) ferrite core-based active sensing method

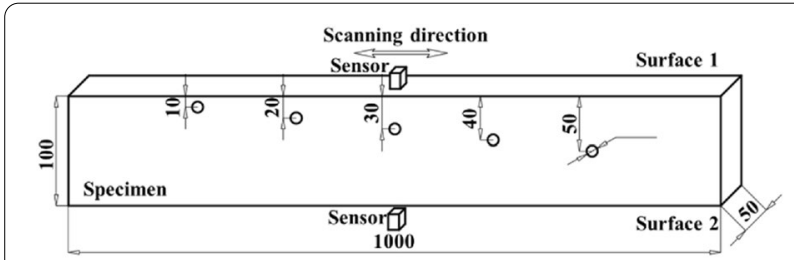

Figure 18 Schematic diagram of MFL testing for deep-buried inner defects

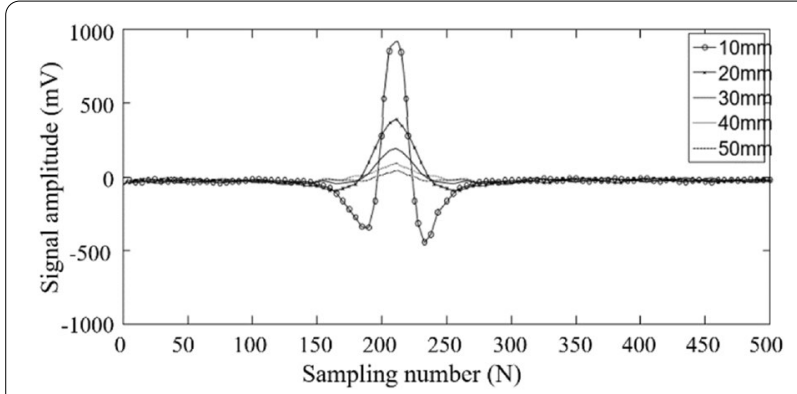

Figure 19 The normal components of the MFL generated by inner defects with buried depths (Sensor scanning above surface 1 indicated in Figure 18)

$1.0 \mathrm{~mm}$; Enameled wire diameter: $0.05 \mathrm{~mm}$; Number of turns: 150). Since the ferrite core has a high permeability, it will generate a strong enhancing effect on MFL course of inner defects, resulting in a greater MFL intensity. In order to illustrate the proposed sensing method, magnetic effect of the ferrite core is simulated, as shown in Figure 17. It can be seen that with the ferrite core, more

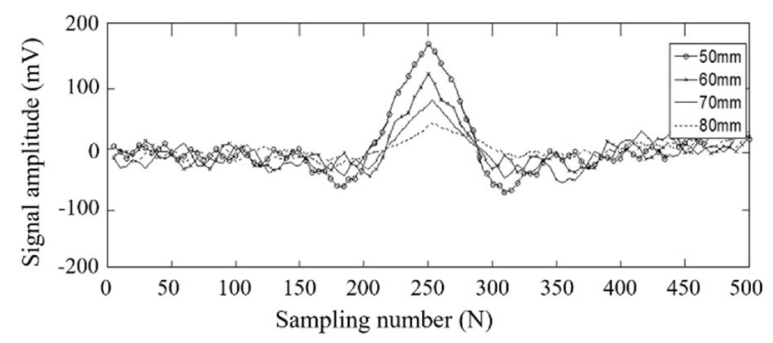

Figure 20 The normal components of the MFL generated by inner defects with buried depths (Sensor scanning above surface 2 indicated in Figure 18)

magnetic flux is guided to leak into the sensor location to enhance the sensitivity.

The experimental scheme for detectable depth of inner defects is designed as schematically illustrated in Figure 18. Five blind holes (Diameter: $2.0 \mathrm{~mm}$; Depth: $20.0 \mathrm{~mm}$ ) with the buried depth changing from $10.0 \mathrm{~mm}$ to $50.0 \mathrm{~mm}$ are made in the steel plate. Turning the steel plate upside-down, when the sensor scans above surface 2, blind holes with the buried depth changing from $50.0 \mathrm{~mm}$ to $90.0 \mathrm{~mm}$ can be tested. Figures 19 and 20 show the normal components of the MFL generated by the inner defects with the proposed sensor placing at the lift-off distance of $1.0 \mathrm{~mm}$ at a fixed motion speed of $50.0 \mathrm{~mm} / \mathrm{s}$. It can be seen that the shape of the obtained MFL signal measured by the coil is an even function with the largest amplitude in the center; in contrast, the MFL signal measured by Hall sensor is an odd function with the sharpest gradient in the center as displayed in Figure 12. The reason is that the output of the induction coil depends on the rate of MFL change while the Hall sensor captured the absolute value of MFL. From Figures 19 and 20 it can be seen that with the buried depth increasing the signal amplitudes and signal noise ratio are both decreasing; however, the inner defect with a buried depth of $80.0 \mathrm{~mm}$ still produces a distinguishing signal, suggesting that proposed MFL method has the potential to detect deeply-buried inner defects and has a promising future in the field of NDT. Besides, there is no doubt that the proposed method has lift-off effect, hence the sensor should be placed as closely as possible to the objects to enhance the detectability for deep-buried inner defects.

\section{Conclusions}

In this paper, the MFL course of inner defects is studied by building magnetic circuit models, performing numerical simulations, and conducting MFL experiments. It is found that when the distance $d_{\mathrm{f}}$ between the defect and sensor is set as a fixed value, the deeper-buried defect 
will produce a greater signal response. Hence, it can be concluded that the near-surface wall has an enhancing effect on the MFL course due to the higher permeability of steel than those of air. Further, a Helmholtz coil magnetization and an active sensing method are proposed to increase the detectable depth limitation of inner defects. Experimental results show that that proposed MFL method has the potential to detect inner defects with the buried depth up to $80.0 \mathrm{~mm}$, which can expand the scope of MFL application. In the future work, the magnetic flux leakage course of overlapped inner defects will be studied.

\section{Authors' Contributions}

JW and WW performed the simulation; EL and YK conducted the experiments; EL and JW analyzed the data; all the authors contributed to the writing and discussion of the paper. All authors read and approved the final manuscript.

\begin{abstract}
Authors' Information
Jianbo Wu, born in 1986, is currently an Associate Professor at School of Mechanical Engineering, Sichuan University, China. He received the B.Sc. degree in mechanism design, manufacturing and automation, M.Sc. degree in precision instruments and mechanics, and the Ph.D. degree in measurement technology and instruments from School of Mechanical Science and Engineering, Huazhong University of Science and Technology, China, in 2009, 2010, and 2014, respectively. His research interests include magnetic flux leakage testing, eddy current thermography, and eddy current testing.
\end{abstract}

Wenqiang Wu, born in 1996, is currently a master candidate at School of Mechanical Engineering, Sichuan University, China. He received his bachelor degree from Sichuan University, China, in 2019

Erlong Li, born in 1989, is currently an assistant researcher at Sichuan University, China. He received the B.Sc. degree in mechanism design, manufacturing and automation, M.Sc. degree in precision instruments and mechanics, and the Ph.D degrees in mechanical and electronic engineering from School of Mechanical Science and Engineering, Huazhong University of Science and Technology, China, in 2012, 2014 and 2018, respectively. His research interests include magnetic flux leakage testing and eddy current testing.

Yihua Kang, born in Hai'an, Jiangsu, China, in 1965. He received the B.Sc., M.Sc., and Ph.D. degrees from School of Mechanical Science and Engineering, Huazhong University of Science and Technology (HUST), China, in 1987, 1990, and 1993, respectively. In 1993, he joined HUST as a Post-Doctoral Fellow, where he was an Adjunct Professor in 1995 and a Professor in 1998. His current research interests include nondestructive testing technology and instrumentation.

\section{Funding}

Supported by National Natural Science Foundation of China (Grant Nos. 51907131, 92060114), and Sichuan Science and Technology Program (Grant Nos. 2020YFG0090, 2021YFG0039, 2020ZDZX0024).

\section{Competing interests}

The authors declare no competing financial interests.

\footnotetext{
Author Details

${ }^{1}$ School of Mechanical Engineering, Sichuan University, Chengdu 610065, China. ${ }^{2}$ School of Mechanical Science and Engineering, Huazhong University of Science and Technology, Wuhan 430074, China.
}

Received: 27 February 2020 Revised: 8 February 2021 Accepted: 25 May 2021

Published online: 12 June 2021

\section{References}

[1] Y Kang, J Wu, Y Sun. The use of magnetic flux leakage testing method and apparatus for steel pipe. Materials Evaluation, 2012, 70(7): 821-827.

[2] F Xu, X Wang, H Wu. Inspection method of cable-stayed bridge using magnetic flux leakage detection: Principle, sensor design, and signal processing. Journal of Mechanical Science and Technology, 2012, 26(3): 661-669.

[3] T A Bubenik, J B Nestlroth, R J Eiber, et al. Magnetic flux leakage (MFL) technology for natural gas pipeline inspection. NDT and E International, 1997, 1(30): 36

[4] Y Ege, M Coramik. A new measurement system using magnetic flux leakage method in pipeline inspection. Measurement, 2018, 123: 163-174.

[5] A Chotzoglou, M Pissas, A. D Zervaki, et al. Visualization of the rolling contact fatigue cracks in rail tracks with a magneto optical sensor. Journal of Nondestructive Evaluation, 2019, 38(3): 68

[6] Y Li, J Wilson, GYTian. Experiment and simulation study of 3D magnetic field sensing for magnetic flux leakage defect characterisation. NDT \& E International, 2007, 40(2): 179-184.

[7] A R Ramírez, J S D Mason, N Pearson. Experimental study to differentiate between top and bottom defects for MFL tank floor inspections. NDT \& E International, 2009, 42(1): 16-21.

[8] K Tsukada, M Yoshioka, Y Kawasaki, et al. Detection of back-side pit on a ferrous plate by magnetic flux leakage method with analyzing magnetic field vector. NDT \& E International, 2010, 43(4): 323-328.

[9] Y Sun, J Wu, B Feng, et al. An opening electric-MFL detector for the NDT of in-service mine hoist wire. IEEE Sensors Journal, 2014, 14(6): 2042-2047

[10] H R Weischdel. The inspection of wire ropes in service: a critical review. Materials Evaluation, 1985, 43(13): 1592-1605.

[11] E Altschuler, A Pignotti. Nonlinear model of flaw detection in steel pipes by magnetic flux leakage. NDT \& E International, 1995, 28(1): $35-40$

[12] Eickemeyer. Magnetic gage for testing the magnetic conductivity of metals. U.S. patent 413,338, 1889

[13] CW Burrows. Method of and apparatus for testing magnetizable objects by magnetic leakage, U.S. patent 1,322,405, 1919.

[14] Y Sun, Y Kang. High-speed magnetic flux leakage technique and apparatus based on orthogonal magnetization for steel pipe. Materials Evaluation, 2010, 68(4): 452-458.

[15] Yanhua S, Yihua K. The feasibility of Omni-directional defects MFL inspection under a unidirectional magnetization. International Journal of Applied Electromagnetics and Mechanics, 2010, 33: 919-925

[16] J Wu, H Fang, X Huang, et al. An online MFL sensing method for steel pipe based on the magnetic guiding effect. Sensors, 2017, 17(12): 2911.

[17] Y Ma, R He, J Chen. A method for improving SNR of drill pipe leakage flux testing signals by means of magnetic concentrating effect. IEEE Transactions on Magnetics, 2015, 51(9): 1-7.

[18] A Joshi, L Udpa, S Udpa, et al. Adaptive wavelets for characterizing magnetic flux leakage signals from pipeline inspection. IEEE Transactions on Magnetics, 2006, 42(10): 3168-3170.

[19] S M Dutta, F H Ghorbel, R K Stanley. Dipole modeling of magnetic flux leakage. IEEE Transactions on Magnetics, 2009, 45(4): 1959-1965.

[20] J Wu, Y Sun, Y Kang, et al. Theoretical analyses of MFL signal affected by discontinuity orientation and sensor-scanning direction. IEEE Transactions on Magnetics, 2014, 51(1): 1-7.

[21] S Yang, Y Sun, L Udpa, et al. 3D simulation of velocity induced fields for nondestructive evaluation application. IEEE Transactions on Magnetics, 1999, 35(3): 1754-1756.

[22] J Wu, Y Sun, B Feng, et al. The effect of motion-induced eddy current on circumferential magnetization in MFL testing for a steel pipe. IEEE Transactions on Magnetics, 2017, 53(7): 1-6.

[23] Y K Shin, W Lord. Numerical modeling of moving probe effects for electromagnetic nondestructive evaluation. IEEE Transactions on Magnetics, 1993, 29(2): 1865-1868

[24] B T Bastian, N Jaspreeth, S K Ranjith, et al. Visual inspection and characterization of external corrosion in pipelines using deep neural network. NDT \& E International, 2019, 107: 102134.

[25] A A Carvalho, J M A Rebello, LV S Sagrilo, et al. MFL signals and artificial neural networks applied to detection and classification of pipe weld defects. NdT \& E International, 2006, 39(8): 661-667. 
[26] A Khodayari-Rostamabad, J P Reilly, N K Nikolova, et al. Machine learning techniques for the analysis of magnetic flux leakage images in pipeline inspection. IEEE Transactions on Magnetics, 2009, 45(8): 3073-3084

[27] ISO. ISO 9402 First edition. Seamless and welded (except submerged arc-welded) steel tubes for pressure purposes- Full peripheral magnetic transducer/flux leakage testing of ferromagnetic steel tubes for the detection of longitudinal imperfection. 1989-07-01.

[28] J Wu, F Hui, L Long, et al. The signal characteristics of rectangular induction coil affected by sensor arrangement and scanning direction in MFL application. International Journal of Applied Electromagnetics and Mechanics, 2016, 52(3-4): 1257-1265.

[29] A Sophian, G Y Tian, S Zairi. Pulsed magnetic flux leakage techniques for crack detection and characterisation. Sensors and Actuators A: Physical, 2006, 125(2): 186-191.
[30] X Lu, G Li, L Chen, et al. Study on low frequency AC excitation magnetization magnetic flux leakage testing for defects with different depths. ASME Pressure Vessels and Piping Conference (PVP 2018), Prague, Czech Republic: JUL 15-20, 2018.

[31] K Tsukada, Y Majima, Y Nakamura, et al. Detection of inner cracks in thick steel plates using unsaturated AC magnetic flux leakage testing with a magnetic resistance gradiometer. IEEE Transactions on Magnetics, 2017, 53(11): 1-5.

[32] J A Parra-Raad, S Roa-Prada. Multi-objective optimization of a magnetic circuit for magnetic flux leakage-type non-destructive testing. Journal of Nondestructive Evaluation, 2016, 35(1): 14.

[33] Jianbo W, Hui F, Jie W, et al. The influence of non-uniform wall thickness on MFL testing for a steel pipe. Insight-Non-Destructive Testing and Condition Monitoring, 2015, 57(12): 703-708.

\section{Submit your manuscript to a SpringerOpen ${ }^{\circ}$ journal and benefit from:}

- Convenient online submission

- Rigorous peer review

- Open access: articles freely available online

- High visibility within the field

- Retaining the copyright to your article

Submit your next manuscript at $\boldsymbol{\nabla}$ springeropen.com 